\title{
La implementación de la Metodología Building Information Modeling (BIM) para edificios existentes en Chile
}

\author{
The implementation of the Building Information Modeling Methodology (BIM) for existing \\ buildings in Chile
}

\author{
Jaime Soto Muñoz \\ Dpto. Ciencias de la Construcción, \\ Universidad del Bío-Bío, Chile, \\ jaimesoto@ubiobio.cl \\ Jesús Pulido Arcas \\ Dpto. Ciencias de la Construcción, \\ Universidad del Bío-Bío, Chile, \\ jpulido@ubiobio.cl
}

\author{
Rodrigo García Alvarado \\ Dpto. Teoría y Diseño de la Arquitectura, \\ Universidad del Bío-Bío, Chile, \\ jpulido@ubiobio.cl \\ Gastón Arias Aravena \\ Esc. Ing. en Construcción, \\ Universidad del Bío-Bío, Chile, \\ gaarias24@gmail.com
}

\begin{abstract}
BIM technology is currently being implemented in the construction industry, though it is still underdeveloped in relation to Facility Management (FC) of extant buildings. There is a strong potential for future development due to the visualization and data analysis capabilities of this technology, amongst others (Becerik-Gerber, Jazizadeh, Li, \& Calis, 2012). This research investigates how BIM can be implemented in existing buildings currently in operation. Using a public facility at the University of Bio-Bio as a case study, conclusions are drawn with respect the capabilities of BIM in order to optimize maintenance and operation of existing buildings.
\end{abstract}

Keywords: Edificios existentes; Modelación; BIM; Operación: Mantención

\section{Introducción}

Building Information Modeling (BIM) está siendo ampliamente adoptado por la industria de la construcción y tiene posibilidades aún sin desarrollar para proporcionar y apoyar las prácticas de Facility Management (FM), gracias a sus funcionalidades de visualización y análisis, entre otras (Becerik-Gerber et al., 2012). De este modo, dichas herramientas pueden ayudar a la implementación de estrategias de operación y mantención, cuyo costo puede ascender hasta a un $70 \%$ del presupuesto total de un proyecto (Hernández Flórez, 2014), siendo "costo" aquel entendido como el sacrificio de un recurso que se asigna para llevar a cabo un objetivo específico (Horngren, Datar, \& Foster, 2007). Por otro lado, el mantenimiento no solo se relaciona con un diseño perfectamente desarrollado, sino que se debe tener en cuenta una implementación periódica del mismo (Mascia \& Sartorti, 2011).

A medida que Chile está desarrollando especialistas e investigadores, BIM se establece como una metodología eficiente de trabajo en edificios nuevos. Estas nuevas construcciones diseñadas por computadora con una modelación en 3D, presentan diferencias notables respecto a los procesos asistidos por computadora (CAD) en 2D. Esto, a la hora de desarrollar un proyecto, exige y mejora bastante la calidad y la cantidad de información que se puede obtener con este método de diseño. Como la operación y el mantenimiento de un edificio impactan más que los procesos de construcción, la relevancia de la sostenibilidad de estos procesos debe ser parte de los criterios actuales. En el país, los principios de sostenibilidad son obligaciones que hoy en día se imponen en el desarrollo de metas no sólo presidenciales y de organismos públicos, sino de forma más imperativa en empresas privadas (CORFO, 2015); el objeto es optimizar la gestión de los proyectos incluyendo además el ciclo de vida del edificio.

Los requisitos exigidos por la metodología BIM exigen que los componentes constructivos sean considerados como entidades (Chávez González \& Francisco, 2015), es decir, constituyen los elementos esenciales de una construcción, los cuales contienen características funcionales y físicas en una representación virtual (Lancharro Cordero, 2015). Esto permite a arquitectos, ingenieros y constructores rastrear, hacer seguimiento y controlar sus proyectos de manera efectiva durante su ciclo de vida. Todo ello representa una gran oportunidad para optimizar y hacer más eficiente la gestión de los recursos en un edificio.

Sin embargo, el desafío surge cuando se plantea qué hacer con los edificios ya existentes. Los gestores de la mayoría de los edificios que conocemos no tienen la oportunidad de aplicar acciones de ahorro de energía, reducción de impacto ambiental, o promover un espacio interior saludable, y solo pueden hacer planes generales de operación y mantención.

El deterioro progresivo de una construcción es consecuencia del uso del mismo. Esta se desgasta desde el mismo momento en que esta comienza a usarse, por lo que es necesario la aplicación de un mantenimiento constante (Gómez Hoyos, 
2013). En este sentido, el estudio presenta una revisión de publicaciones recientes sobre el tema y un estudio de caso, basado en un edificio del año 2011, que incluye instalaciones de acondicionamiento ambiental y sistemas constructivos para un uso eficiente de la energía. Los resultados muestran que la implementación de BIM es escasa en edificios existentes, incluso en los más recientes, y que existen dificultades añadidas a las que hay que enfrentarse a la hora de "BIM-izar" una construcción ya en uso. Ante esto, el estudio analiza la aplicación de la metodología BIM en edificios existentes, la que se enfrenta a retos diferentes, relacionados principalmente con la naturaleza multidisciplinar del intercambio de información.

\section{Definición de Modelación de Información de Edificios (BIM)}

La modelación de información de un edificio es una de las áreas importantes en la investigación actual de la realidad virtual y considera no sólo el desarrollo tecnológico, como parte importante de los estudios, sino también se refiere a la aplicación de las tecnologías y su adopción por parte de los profesionales del área (Gu \& Kerry, 2010). El modelo de información de un edificio se puede definir por la norma internacional ISO 29481-1 como "representación digital compartida de las características físicas y funcionales de cualquier objeto construido [...] que constituye una base fiable para las decisiones" (ISO 29481-1:2016 - Building information models -- Information delivery manual -- Part 1: Methodology and format, 2016). Esta definición, se suma al principio que indica que BIM se origina a partir de modelos de productos (Cerovsek, 2011) y que se aplica ampliamente en distintas áreas productivas (Wong, Johnny \& Yang, 2010). Por lo que también se utiliza en distintos tipos de proyectos de edificación (Eastman, Teicholz, Sacks, \& Liston, 2011).

Esta metodología se implementa mediante softwares orientados a distintos tipos de objetos, con el fin de parametrizar y representar componentes de una edificación. Los objetos pueden tener atributos geométricos o no tenerlos, además de información funcional, semántica o topológica; de forma integrada, todos ellos son parte de un proceso de generación, planificación y gestión de datos de un edificio a lo largo de un ciclo de vida.

Para poner en prá la metodología BIM es necesario disponer de diversas herramientas; primeramente se debe decidir qué proveedor será el principal para lo cual el mercado ofrece una variada oferta: Revit (Autodesk), Graphisoft (Archicad), Nemetschek (Allplan), etc (Oya Sala, 2015). Actualmente las plataformas BIM más usadas en Chile son Revit y Naviswork (Autodesk), seguida de Archicad (Graphisoft). (Loyola, 2016)

Cuando llega el momento de elegir una herramienta de modelado es necesario tener en cuenta ciertos parámetros. Uno de los más importantes es la interoperabilidad entre las distintas herramientas de la metodología BIM y la herramienta de modelado que se utilizará. Este punto es especialmente importante, de modo que se pueda integrar la información al proyecto y administrarla de la mejor manera posible (Ramírez Castro, 2015).

\section{Mantenimiento y Operación de una Edificación.}

La correcta definición de mantenimiento es la que establece que es el "conjunto de acciones, operaciones y actitudes que permiten mantener o reestablecer un bien en un estado específico, o en la medida de asegurar un servicio determinado" (Olivares Sánchez, 2015). La implementación de la metodología BIM ayuda a gestionar el mantenimiento de un edificio durante su ciclo de vida; en el país esto se realiza mediante los planos As-built o planos conforme a obra. El propósito es poder gestionar el proceso de mantenimiento de equipos e instalaciones en una condición particular o volverlos a dicha condición (Prando, 1996). Así, se puede diferenciar en mantenimiento preventivo y el mantenimiento correctivo. El primero se relaciona con acciones llevadas a cabo periódicamente con el fin de prevenir una falla así permitiendo el uso de la edificación de forma normal; el segundo tipo de mantenimiento, "correctivo y/o reactivo", ocurre en una construcción cuando se produzca una falla y esta requiere de reparación para continuar con la funcionalidad del edificio (Olarte, Botero \& Cañon, 2010).

Por otra parte, la operación puede relacionarse con la acción de llevar a cabo algo, y desde el punto de vista de una edificación esta operación se relaciona con el funcionamiento esperado del edificio. Estas dos acciones (mantenimiento y operación) pueden ser medidas en función de un costo asociado, el cual se puede definir como: "valor monetario de los recursos que se entregan o prometen entregar a cambio de bienes o servicios que se adquieren" (García Colín, 2014). Una definición alternativa puede ser: "Sacrificio de recursos que se asigna para lograr un objetivo específico. Un costo por lo general se mide como la cantidad monetaria que debe pagarse para adquirir un adquirir bienes o servicios" (Horngren et al., 2007).

La necesidad de desarrollar un plan de mantenimiento es principalmente la búsqueda de la eficiencia de recursos. Emerson Process Management, en un artículo publicado el 2003, plantea lo siguiente: "Una estrategia de mantenimiento preventivo asume que el equipo es relativamente confiable hasta que, después de algún período de tiempo, entra a una zona de 'desgaste' donde las fallas aumentan. Para posponer este desgaste, se da servicio al equipo basado en el calendario o en tiempo de corrida - sin importar si lo necesita o no. En promedio, este enfoque de 'corríjalo por si acaso' es aproximadamente $30 \%$ menos costoso que el mantenimiento reactivo" (Emerson Process Management, 2003).

Esto revela la importancia que presenta un plan de mantenimiento preventivo, en contraposición a la realización de un mantenimiento meramente reactivo o correctivo. Por otra parte, en la gestión del mantenimiento pueden definirse 3 responsabilidades atribuibles al mantenimiento (Tavares, 2004):

- Reducción del tiempo de paralización de los equipos que afectan la operación.

- Reparación, en el tiempo oportuno, de los daños que reducen el potencial de ejecución de servicios. 
- Garantía de funcionamiento de las instalaciones, de manera que los productos o servicios satisfagan criterios establecidos por el control de calidad y estándares preestablecidos.

Con estas responsabilidades, es posible entender los principales objetivos que se deben establecer a la hora de desarrollar un plan de mantenimiento.

\section{Facility Management.}

Una buena gestión del mantenimiento de un edificio considera el ciclo de vida de cada elemento, asegura el correcto funcionamiento de los mismo a la vez que tiene en cuenta una mayor eficiencia en los costos (Viveros, Stegmaier, Kristjanpoller, Barbera, \& Crespo, 2013). En consecuencia, se ha probado que un buen plan de mantenimiento permite un ahorro sustancial de los costos de Facility Management (FM) al mismo tiempo que mejora los servicios que la edificación ofrece a sus usuarios (Collado, 2009)

En la actualidad si se hace referencia a mantenimiento, operación y gestión de bienes inmuebles, se habla de Facility Management (FM) (Hernández Flórez, 2014). Esta disciplina trata todo lo relacionado con la gestión de las edificaciones en uso. La agencia International Facility Management Association (2017) lo define como: "Una profesión que abarca la disciplina múltiple para asegurar la funcionalidad del entorno construido mediante la integración de las personas, el lugar, los procesos y la tecnología". Esta disciplina está siendo muy estudiada y además, está siendo recocida como un punto importante en las grandes empresas. C. Hodges, en un artículo para una revista de Facilities Management entrega la siguiente conclusión: "Durante muchos años, sobre todo en los EE.UU., se ha hecho hincapié en la reducción del costo inicial de las instalaciones, por lo que el énfasis ha estado en el porcentaje más bajo de costo de existencia de una organización. Pronto, los interesados se darán cuenta de que hay muchos más dólares en juego que pueden ser conducidos a la línea de fondo, si se hace hincapié en las mejoras de productividad y eficiencia de operación y mantenimiento" (Thomas - Mobley \& Khuncumchoo, 2006). Estos conceptos ayudan a entender la importancia que tiene el desarrollo de un plan de gestión de edificaciones, y lo ejecutivo de su aplicación, considerando que los costos asociados a la etapa de operación de una construcción son muy altos, comparados con los costos que se incurren en el diseño y construcción. Así mismo, reevalúa el papel y contribución potencial de la aplicación de BIM en edificios existentes.

Adicionalmente, los beneficios tiene la disciplina de FM son variadas. Así lo afirma Lavy en un estudio de 2008 sobre construcciones educacionales: "No sólo puede mejorar el rendimiento físico y la apariencia del edificio y sus sistemas, sino que también puede aumentar el nivel de satisfacción que los usuarios sienten durante su estancia / trabajo / enseñanza / aprendizaje en ese edificio, y mejorar la eficiencia con la que el edificio es mantenido y operado". De acuerdo a esta investigación, es posible incluir el FM en muchas funciones, que van desde la planificación estratégica y la gestión de activos, pasando por la planificación, el diseño, la gestión de la construcción, operación, mantenimiento y servicios administrativos generales.

A medida que los clientes comienzan a tener estas necesidades, el potencial de los servicios de gestión de las edificaciones en uso se expande, ofreciendo nuevas e importantes oportunidades de negocio para aquellas empresas de arquitectura que tengan, o deseen, desarrollar unas habilidades apropiadas (Nävy, 2006); en este punto, es necesario destacar que el FM engloba la gestión de todos los elementos, personas, actividades y recursos en función de la eficiencia de la etapa de operación y mantenimiento.

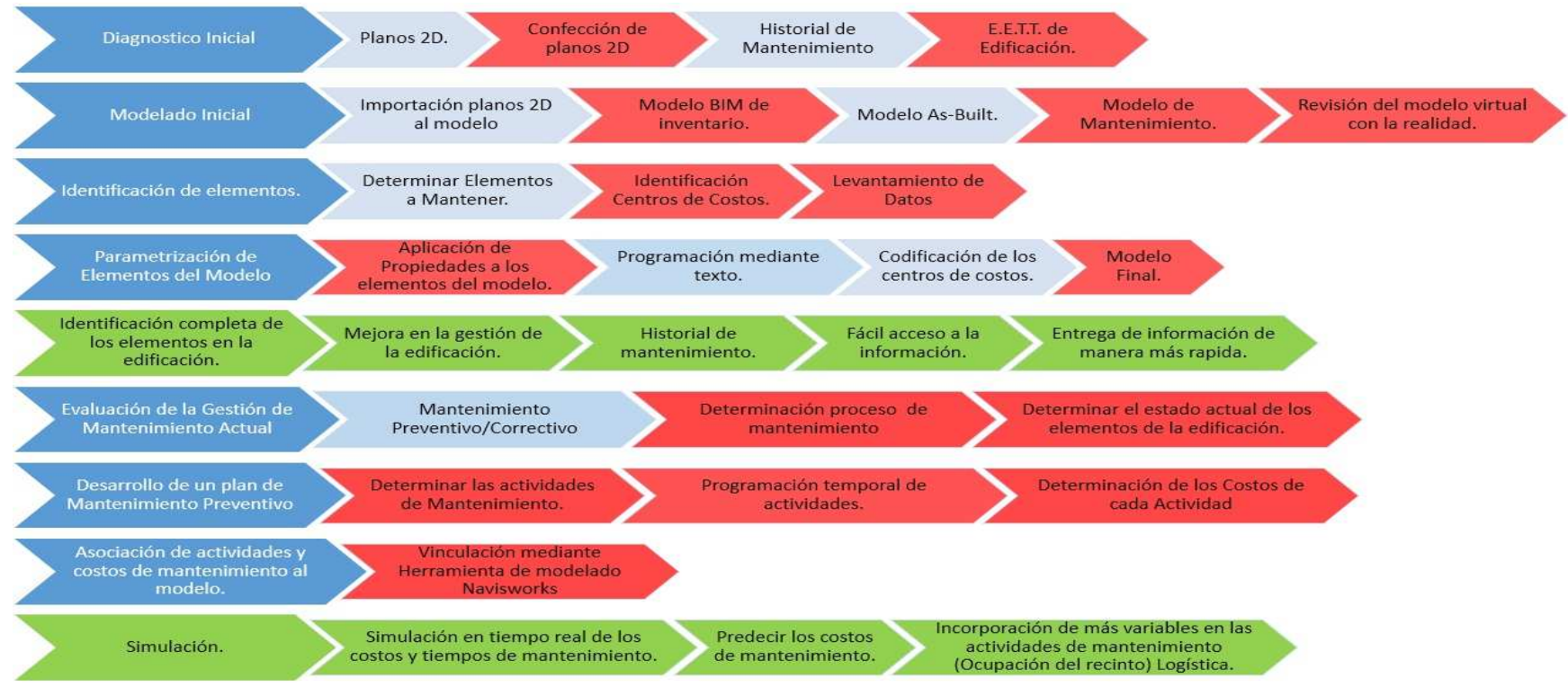

Figura 1. Proceso de recogida de datos y BIM-ización del edificio (elaboración propia) 
Estos datos contrastan, sin embargo, con la realidad a nivel nacional, donde el BIM encuentra un difícil acomodo en este campo. Dentro de los profesionales que usan exclusivamente BIM en su trabajo (22\%), el mayor porcentaje de los mismo que usa el BIM solamente en la fase de diseño es de un $47 \%$, en contraste con el escaso $7 \%$ que lo usa en la etapa de FM; un $69 \%$ de los mismos reconoce que nunca ha usado BIM para gestionar el mantenimiento de un edificio existente (Loyola, 2016).

\section{Proceso de "BIM-ización de edificios"}

Conscientes de la necesidad de avanzar en la implantación de BIM, y de que existe un vacío en su aplicación en el FM de edificios existentes, este trabajo propone una metodología para recopilar datos de un edificio construido e implementarlos en un entorno BIM con el objeto de gestionar su mantenimiento y optimizar los costos. A este proceso se le ha denominado "BIM-izar" el edificio, como un particular aplicación al contexto de las construcciones en el país.

El caso de estudio lo constituye un edificio cuyo uso es administrativo y educacional: El Centro de Investigación en Tecnologías de la Construcción de la Universidad del Bío-Bío (CITEC-UBB) (Figura 2); construido en 2010, lleva en operación 7 años. Tiene una superficie de aproximadamente $600 \mathrm{~m}^{2}$ distribuidos en dos plantas.

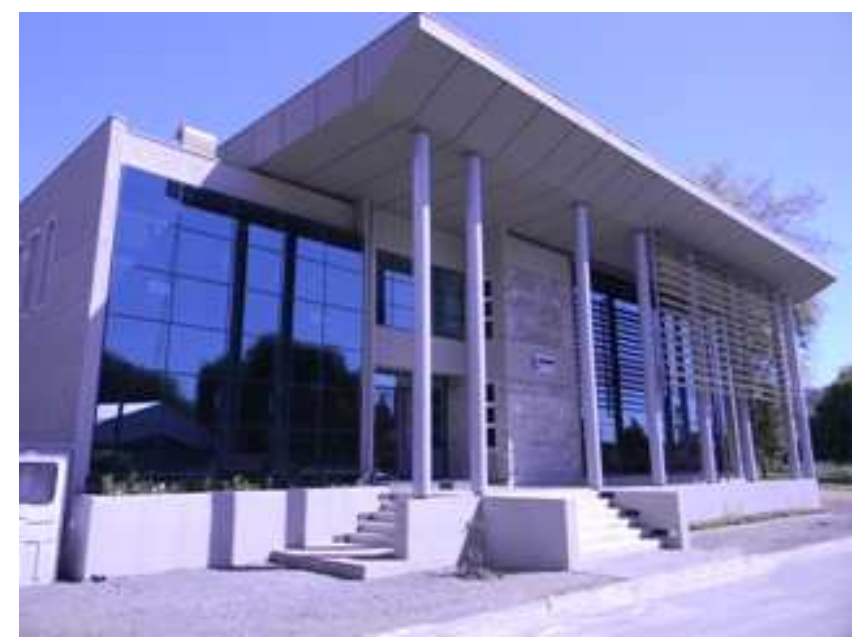

Figura 2: Edificio caso de studio: CITEC UBB (www.citecubb.cl)

Los principales pasos del proceso están resumidos en la Figura 1. De acuerdo a la experiencia obtenida se puede considera el siguiente esquema de trabajo.

\section{Diagnóstico inicial, cuando se dispone de planos en 2D}

Si se dispone de los planos digitalizados, se puede establecer que existe un camino conocido para lograr modelar el edificio. Se utilizan los archivos CAD, para conformar y parametrizar el nuevo trabajo. Se debe generar una validación del estado de conservación y rehabilitaciones practicadas al edificio, lo que debe quedar reflejado tal cual como se encuentra en operación.

\section{Modelado inicial}

Si el edificio dispone de un modelo BIM, el trabajo en esta fase consistirá en corregir aquellos elementos que difieran entre el modelo "as-built" del edificio y lo existente realmente. Se entiende "as-built" como la representación gráfica presentado por un contratista al finalizar un proyecto y que refleja todos los cambios realizados en las especificaciones y los planos de trabajo durante el proceso de construcción. Debe mostrar las dimensiones exactas, la geometría y la ubicación de todos los elementos del trabajo completado bajo el contrato. Si no fuese así, el edificio debe modelarse parcialmente o por completo, según corresponde. En este caso de estudio la modelación realizada fue completa. En este paso, fue importante decidir el nivel del detalle del modelo; se optó por un nivel LOD 300 ó 400 , dependiendo de los elementos. El modelo resultante se denomina "modelo BIM de inventario", que es una perfección de la versión original de CAD. Sin embargo, la precaución está en el proveedor de software, ya que si bien se ha ido desarrollando productos en términos de gráficos, hay excepciones donde no se puede disponer de un inventario acabado para todos los elementos del edificio. El efecto es no tener un elemento del inventario como base gráfica para la gestión de instalaciones FM.

\section{Identificación y parametrización de los elementos del modelo}

A cada elemento del edificio se le asignaron unos parámetros relativos a su costo de mantenimiento, mediante una codificación en formato de texto directamente embebida en la plataforma BIM. Los parámetros se definieron en formato texto, asegurando además su interoperabilidad con otras plataformas como MS Project $\AA$ y Naviswork $\AA$. Finalmente, toda la información quedó incluida en el modelo BIM del edificio. La dificultad está en una gestión eficiente, FM requiere tomar decisiones inteligentes basada en sistemas contables detallados, esto es, cuentas contables que permitan separar elementos del inventario por familias y/o centros de costos asociados a la parámetros de operación y mantenimiento del edificio.

\section{Evaluación de la gestión del mantenimiento actual}

De acuerdo a los planes de mantenimiento del edificio, los cuales fueron proporcionados por el Departamento de Mantenimiento de la Universidad del Bío-Bío, se pudo determinar que el mantenimiento es sólo de tipo correctivo. El proceso habitual consiste en informar mediante correo electrónico al Departamento cuando un elemento falla, el cual introduce los datos en una planilla y le asigna un centro de costo; tras una visita de inspección, se decide la acción a ejecutar, se presupuesta y se lleva a cabo. De acuerdo a los datos disponibles, el costo total de mantenimiento del edificio para el período de un año (2014-2015) asciende a \$551.401. En este sentido, este trabajo pudo observar una debilidad, ya que los centros de costos asignados al caso de estudio eran más bien generales. Lo que sucede es común en los presupuestos de edificios existentes de las instituciones públicas y en algunas edificaciones de mandante privado del país. Las asignaciones para un mantenimiento preventivo son escasos, y se llega más fácil a un inversión costosa, ya que el sistema propende a la inversión en infraestructura. Salvo 
excepciones, no hay criterios que permitan extender la vida útil de la construcción con operaciones eficaces y aprobación de pequeños montos de mantenimiento preventivo.

\section{Desarrollo de un plan de mantenimiento preventivo en BIM (modelo 4D) y asociación de actividades y costos}

Una vez que se conoce por una parte, la situación actual del mantenimiento del edificio y, por otra un modelo BIM de la construcción, modelado a partir de la información existente, se procede a implementar un plan de mantenimiento en el edificio. Para logarlo, se toma como base un modelo BIM 4D, que incluye no sólo todas las propiedades $3 \mathrm{D}$ de los elementos, sino su evolución temporal, logrando de este modo el mencionado 4D. Se debe incluir información, para cada elemento, de su unidad de medida, su cubicación, su período de servicio más probable, su momento de falla más probable, y los costes unitarios de mantenimiento preventivo $y$ correctivo. Esto es, un sistema que tenga en cuenta:

- Reparar desgastes y mantener todos los sistemas existentes

- Responder a las quejas de confort de los ocupantes

- Ejecutar mantenimiento preventivo

- Elaborar procedimientos de acción

Puesto que el objetivo del modelo es optimizar los procesos de FM en los edificios, se valorizó además el coste del desarrollo e implementación del plan. Los costes asociados, incluyendo las horas / hombre de trabajo, se han calculado para este caso de estudio en $\$ 78.809$ por metro cuadrado.

\section{Simulación de los costes de mantenimiento del edificio}

Disponiendo de todos los datos anteriores, se puede simular el deterioro del edificio en un plazo de tiempo determinado usando el modelo BIM 4D y previendo, con un grado de certeza aceptable, el coste de los mantenimiento preventivo y correctivo. Las simulaciones se pueden hacer para varias hipótesis (peor, mejor y más probable), de modo que se disponga de una horquilla presupuestaria de perfil probabilístico más flexible asociada a los costos de mantenimiento. No se recomienda criterios determinísticos puros, ya que aunque se suponen conocidos datos históricos del edificio con certeza, la ocupación de un edificio depende de las personas que habitan en él y estos cambian a través del tiempo.

\section{Incógnitas en el proceso de trabajo.}

La principal dificultad que se encontró al proponer y aplicar esta metodología al caso de estudio fue el incertidumbre en conocer los sistemas constructivos del edificio, de modo que se debía comprobar que todos los datos recogidos tuviesen su correspondiente respaldo técnico existente.

Las visitas de inspección o el uso los escáneres no registran necesariamente la especificación técnica de las partidas que componen el edificio existente. En estos casos se puede realizar ensayos in-situ para determinar algunas de sus propiedades. Sin embargo, esto no asegura la veracidad de los resultados, siendo además muy costoso.

\section{Conclusiones}

Los resultados obtenidos en este caso de estudio se podrán considerar satisfactorios, dado que la implementación del modelo 4D genera un coste completamente asumible si se compara con el costo anual de mantenimiento del edificio. Estas conclusiones coinciden con los hallazgos de otros autores (Eastman et al., 2011) (Building Smart Spanish Chapter \& Rodríguez Castells, 2014).

También es importante señalar la importancia de encontrar metodologías que permitan a los edificios construidos con anterioridad a la aparición de BIM, o construidos recientemente pero sin implementar esta metodología, adaptarse o, en otras palabras, BIM-izarse. De no ser así, se podría crear un dicotomía en la industria de la edificación, distinguiendo entre edificios "pre-BIM", sin posibilidad de recuperación y adaptación a la nueva tecnología, y edificios "post-BIM".

Las conclusiones de este caso de estudio muestran que BIM es perfectamente aplicable a edificios "pre-BIM", y que la aplicación de esta metodología se debe centrar en optimizar su mantenimiento, disminuyendo los costos, alargando su vida útil $\mathrm{y}$, en otras palabras, creando edificios resilientes que puedan absorber nuevas tecnologías.

\section{Agradecimientos}

Los autores desean expresar su agradecimiento a:

Grupo de Investigación en Gestión y Diseño Integrado de Edificios de la Universidad del Bío-Bío.

Proyecto FONDECYT Regular 1171108: Optimization of project delivery processes in public constructions, through the use of Discrete Event Simulation and Building Information Modeling, under an Extreme Collaboration environment.

\section{Referencias}

Becerik-Gerber, B., Jazizadeh, F., Li, N., \& Calis, G. (2012). Application Areas and Data Requirements for BIM-Enabled Facilities Management. Journal of Construction Engineering and Management, 138(3), 431-442. http://doi.org/10.1061/(ASCE)CO.1943-7862.0000433

Building Smart Spanish Chapter, N., \& Rodríguez Castells, R. L. (2014). Spanish journal of BIM : building smart. Spanish Journal of Building Information Modeling, ISSN-e 2386-5784, No. 15, 2, 2015, págs. 4-8. Building Smart Spanish Chapter. Retrieved from https://dialnet.unirioja.es/servlet/articulo?codigo $=5501132$

Cerovsek, T. (2011). A review and outlook for a "Building Information Model" (BIM): A multi-standpoint framework for technological development. Advanced Engineering Informatics, 25(2), 224-244. http://doi.org/10.1016/j.aei.2010.06.003

Chávez González, J. F., \& Francisco, J. (2015). Automatización de las mediciones y presupuestos de los proyectos de edificación con la metodología BIM. Universidad de La Laguna. Retrieved from https://riull.ull.es/xmlui/handle/915/1070 
Collado, M. J. S. (2009). El mantenimiento de un edificio inteligente. B Universidad Panamericana. Retrieved from https://books.google.cl/books?id=_Jt9nQAACAAJ

CORFO. (2015). PEN. Productividad y Construcción Sustentable. Santiago. Retrieved from http://www.agendaproductividad.cl/wpcontent/uploads/sites/22/2014/10/PPT_Programa_Estrategico_C onstruccion_Sustentable.pdf

Eastman, C., Teicholz, P., Sacks, R., \& Liston, K. (2011). BIM Handbook: A Guide to Building Information Modeling for Owners, Managers, Designers, Engineers, and Contractors (2nd ed.). Hoboken, New Jersey: Wiley.

Emerson Process Management (2003) White paper: Reducción de Costos de Operación y de Mantenimiento PlantWeb ® Reducción de costos de operación y de mantenimiento. Retrieved from http://www2.emersonprocess.com/siteadmincenter/PM\%20Centr al\%20Web\%20Documents/EspanolPlantWeb-ops-maint.pdf

García Colín, J. (2014) Contabilidad de costos. Mcgraw-hill Education. 4 ta edic. Mexico.

Gómez Hoyos, P. C. (2013). Elaboración del Plan Operativo, Análisis de Costos y Presupuesto, en el mantenimiento de la infraestructura de la ESPE-L para el período 2013 - 2016. LATACUNGA / ESPE / 2013. Retrieved from http://repositorio.espe.edu.ec/xmlui/handle/21000/7181

Hernández Flórez, J. G. (2014). Metodología para evaluar un sistema de mantenimiento efectivo basado en Facility Management para un centro hospitalario. Retrieved from https://repository.eafit.edu.co/handle/10784/2904\#.WWa05Yq1u Rs

Horngren, C. T., Datar, S. M., \& Foster, G. (2007). Contabilidad de costos : un enfoque gerencial. Pearson Educacion.

IFMA: International Facility Management Association (2017) What is Facility Management?. Retrieved from https://www.ifma.org/about/what-is-facility-management

ISO 29481-1:2016 - Building information models -- Information delivery manual -- Part 1: Methodology and format. (2016). Retrieved from https://www.iso.org/standard/60553.html

Lancharro Cordero, L. (2015). BIM, la metodología de trabajo que nos acecha. Técnica Industrial, 312, 68-71. Retrieved from https://dialnet.unirioja.es/servlet/articulo?codigo $=5367184$

Lavy, S. (2008). Facility management practices in higher education buildings. Journal of Facilities Management, 6(4), 303-315. http://doi.org/10.1108/14725960810908163

Loyola, M. (2016). Encuesta Nacional BIM 2016: Informe de Resultados, 1-13. Retrieved from http://www.bim.uchilefau.cl/

Mascia, N. T., \& Sartorti, A. L. (2011). Identificación y análisis de patologías en puentes de carreteras urbanas y rurales. Revista Ingeniería de Construcción, 26(1), 05-24. http://doi.org/10.4067/S0718-50732011000100001
Nävy, J. (2006). Facility management: Grundlagen Computerunterstützung Systemeinführung Anwendungsbeispiele. Springer.

Gu, N. \& London, K. (2010) Understanding and facilitating BIM adoption in the AEC industry. Automation in ConstructionVolume 19 , Issue 8 , p.988-999

Olarte, W.; Botero, M.; Cañon B.(2010) Técnicas de mantenimiento predictivo utilizadas en la industria. Scientia Et Technica, XVI(45), 223-226. Retrieved from http://www.redalyc.org/articulo.oa?id=84917249041

Olivares Sánchez, A. (2015). Mantenimiento integral de edificios e instalaciones: análisis y medidas de mejora. Escuela Técnica Superior de Ingeniería Industrial. Universidad de Cartagena. Retrieved from http://repositorio.upct.es/handle/10317/4939

Oya Sala, T. (2015). Impacto del BIM en la gestión del proyecto y la obra de arquitectura: Un proyecto con REVIT. Retrieved from https://riunet.upv.es/handle/10251/55227

Prando, R. R. (1996). Manual de gestión de mantenimiento a la medida. Retrieved from https://www.revistavirtualpro.com/biblioteca/manual-de-gestionde-mantenimiento-a-la-medida

Ramírez Castro, J. (2015). Desarrollo de un Proyecto de Construcción con la tecnología BIM: Centro Multiusos de Llíria. Universidad Politécnica de Valencia. Retrieved from https://riunet.upv.es/handle/10251/48947?show=full

Tavares, L. (2004). Administracion Moderna de Mantenimiento. Editorial Interamericana S.A.

Thomas-Mobley, L., \& Khuncumchoo, N. (2006). A facility manager's approach to standardized construction contracts. Journal of Facilities Management, 4(4), 234-244. http://doi.org/10.1108/14725960610702929

Viveros, P., Stegmaier, R., Kristjanpoller, F., Barbera, L., \& Crespo, A. (2013). Propuesta de un modelo de gestión de mantenimiento y sus principales herramientas de apoyo. Ingeniare. Revista Chilena de Ingeniería, 21(1), 125-138. http://doi.org/10.4067/S071833052013000100011

Wong, Johnny \& Yang, J. (2010). Research and application of Building Information Modelling (BIM) in the Architecture, Engineering and Construction (AEC) industry: a review and direction for future research. In Proceedings of the 6th International Conference on Innovation in Architecture, Engineering \& Construction (AEC) (pp. 356-365). Loughborough UNiversity, UK: Pennsylvania State UNiversity. Retrieved from https://eprints.qut.edu.au/38333/ 